\title{
Boundedness of solutions of forced isochronous oscillators with singularity at resonance
}

Haiyong Wang ${ }^{1}$ and Shunjun Jiang ${ }^{2^{*}}$

\section{"Correspondence:}

jiangshunjun@njut.edu.cn

${ }^{2}$ College of Sciences, Nanjing

University of Technology, Nanjing,

210009, People's Republic of China

Full list of author information is

available at the end of the article

\begin{abstract}
In this paper we study the second-order periodic system: $x^{\prime \prime}+V^{\prime}(x)+p(t) g(x)=F_{x}(x, t)$ where $V(x)$ has a singularity. Under some assumptions on the $V, F, g$, and $p$, by Ortega's small twist theorem, we obtain the existence of quasi-periodic solutions and boundedness of all the solutions.
\end{abstract}

Keywords: isochronous oscillators; repulsive singularity; small twist theorem; quasi-periodic solutions; boundedness of solution

\section{Introduction and main result}

The question of boundedness of all solutions of

$$
x^{\prime \prime}+V^{\prime}(x, t)=0
$$

initiated by Littlewood [1], has been the subject of numerous studies. Morris [2] has shown that when $V^{\prime}(x, t)=x^{3}-p(t)$, where $p(t)$ is continuous, all solutions are bounded. Levi [3] considered (1.1), where the potential $V$ satisfies a superquadratic growth and has a singularity. The author reached a similar conclusion as in [1]. In [4], Liu has proved boundedness of all the solutions of the following equation with an asymmetric nonlinearity

$$
x^{\prime \prime}+\alpha x^{+}-\beta x^{-}=p(t)
$$

where $x^{+}=\max \{x, 0\}, x^{-}=\max \{-x, 0\}, \alpha, \beta>0$. For this case, all the solutions of the unperturbed equation

$$
x^{\prime \prime}+\alpha x^{+}-\beta x^{-}=0,
$$

have a common period $T(1 / \sqrt{a}+1 / \sqrt{b})$, which means that (1.2) is isochronous.

In [5], Bonheure et al. study the following equation, which is a perturbation of the isochronous oscillator:

$$
x^{\prime \prime}+V^{\prime}(x)+g(x)-p(t)=0,
$$


where $V(x)$, defined on $(a,+\infty)$, has a repulsive singularity and satisfies

$$
\lim _{n \rightarrow+\infty} \frac{2 V(x)}{x^{2}}=\frac{m^{2}}{4}, \quad \lim _{n \rightarrow a_{+}} V(x)=+\infty
$$

$m$ is a positive integer, $a \in(-\infty, 0)$. The perturbation $g(x)-p(t)$ satisfies the LazerLandsman condition

$$
4 g^{+}>\max _{\theta} p_{*}(\theta)
$$

where the function $g(x)$ is smooth and bounded, $\lim _{n \rightarrow+\infty} g(x)=g^{+}, p_{*}(\theta)=\int_{0}^{2 \pi} p(t+$ $\theta)|\sin (m t / 2)| d t$ and $p(t)=p(t+2 \pi)$. The authors assume that all solutions of the unperturbed equation

$$
x^{\prime \prime}+V^{\prime}(x)=0
$$

are $2 \pi / m$, which means that (1.4) is an isochronous oscillator with period $2 \pi / m$. The authors proved the existence of $2 \pi$-periodic solutions of (1.3). We refer for more details on the isochronous system to [5] and the references therein.

Recently, Liu [6] studied the quasi-periodic solutions and boundedness of all solutions of the isochronous oscillators equation (1.3) where $V(x)$ has a singularity, the nonlinearity $g(x)$ is a bounded perturbation, and $p(t)$ is $2 \pi$-periodic. Moreover, the following assumptions hold:

(1) $V(0)=V^{\prime}(0)=0, V^{\prime \prime}(x)>0$ for $x \neq 0$ and $\lim _{x \rightarrow a_{+}} V(x)=+\infty, a \in(-\infty, 0)$, and $V(x)$ is defined on $(a,+\infty)$.

(2) The function

$$
W(x):=\frac{V(x)}{V^{\prime}(x)}
$$

is smooth in $(-1, \infty)$ and the $\operatorname{limit}_{\lim _{x \rightarrow-1}} W(x)$ exists. Furthermore, the following estimates hold: for each $1 \leq k \leq 6$ there is a constant $c_{0}$ such that

$$
|W(x)| \leq c_{0}(1+x), \quad\left|W^{k}(x)\right| \leq c_{0}, \quad \text { for } x \in[-1, \infty)
$$

(3) The positive function $V$ is smooth and, for $0 \leq k \leq 6$,

$$
\left|(1+x)^{k} V^{k}(x)\right| \leq c_{0}^{\prime} V(x)
$$

where $c_{0}^{\prime}$ is a positive constant.

(4) For $x>0$, let $\Phi(x)=V(x)-\frac{1}{8} x^{2}$; the function $\Phi$ satisfies

$$
\lim _{x \rightarrow+\infty} x^{k} \Phi^{k}(x)=0,
$$

for every positive integer $k$. By the results in [5], the auxiliary autonomous system $x^{\prime \prime}+V^{\prime}(x)=0$ is an isochronous system with period $T=2 \pi$. 
(5) The function $g$ is bounded on the interval $[-1,+\infty)$ and $g(x)>0$ for $x>0$. Moreover, the following inequalities hold:

$$
\lim _{x \rightarrow+\infty}(1+x)^{k} \frac{d^{k}}{d x^{k}} g(x)=0 .
$$

(6) If $\operatorname{limit}_{\lim _{x \rightarrow+\infty}} g(x)=g^{+}$exists and $p^{*}(\theta)=\int_{0}^{2 \pi} p(t+\theta)|\sin (t / 2)| d t$, the following condition of Lazer-Landesman type holds:

$$
4 g^{+}>\max _{\theta} p^{*}(\theta)
$$

Liu first reduced the system to a normal form and then applied a variant of Moser's twist theorem of invariant curves to prove the existence of quasi-periodic solution and the boundedness of all solutions. This result relies on the fact that the nonlinearity can guarantee the twist condition of the KAM theorem. The assumptions (5) and (6) satisfy the Lazer-Landesman condition which plays a key role in the boundedness problem. In fact, it has been shown by Alonso and Ortega [7] that, when the Lazer-Landesman condition cannot be satisfied, the solutions with large initial conditions are unbounded either in the past or in the future.

We observe that in [5, 6], the perturbation $g(x)-p(t)$ is smooth and bounded, so a natural question is to find sufficient conditions on $g(x)$ and $p(t)$ such that all solutions of (1.3) are bounded when the perturbation is unbounded. The purpose of this paper is to deal with this problem. We can refer to more papers on the Littlewood Problem on unbounded perturbation such as $[8,9]$.

Motivated by the papers [5, 6], we consider the following equation:

$$
x^{\prime \prime}+V^{\prime}(x)+p(t) g(x)=F_{x}(x, t)
$$

where $0<\alpha<1$. We suppose that (1)-(4) hold; moreover, we have

(5) $F(x, t) \in C^{7,6}\left(S^{1} \times R\right)$ and

$$
\lim _{x \rightarrow+\infty} \frac{F(x, t)}{|x|^{\alpha} x}=0, \quad F(x, t+2 \pi)=F(x, t) .
$$

$\left(6^{\prime}\right) p(t)=p(t+2 \pi)$ and

$$
\lim _{x \rightarrow+\infty} \frac{g(x)}{|x|^{\alpha}}=1, \quad G(x)=\int_{0}^{x} g(s) d s
$$

where $0<\alpha<1$.

$\left(7^{\prime}\right)$ We mention a condition similar to the Lazer-Landesman condition: $\int_{0}^{2 \pi} \sin \frac{\theta}{2} \times$ $\left|\sin \frac{\theta}{2}\right|^{\alpha} p\left(t_{0}+\theta\right) d \theta>0$.

Our main result is the following theorem.

Theorem 1 Suppose the assumptions (1)-(4) and (5')-(7') hold, then (1.5) has infinitely many quasi-periodic solutions and all the solutions of (1.5) are bounded. 
The main idea of our proof is acquired from [6]. The proof of Theorem 1 is based on the small twist theorem due to Ortega [10]. It mainly consists of two steps. The first one is to transform (1.5) into a perturbation a integrable Hamilton system. The second one is to show that the Poincaré map of the equivalent system satisfies Ortega's twist theorem, then the desired result can be obtained.

\section{The proof of the theorem}

\subsection{Action-angle variables}

Observe that (1.5) is equivalent to the following Hamiltonian system:

$$
x^{\prime}=\frac{\partial H}{\partial x}, \quad y^{\prime}=-\frac{\partial H}{\partial x}
$$

with the Hamiltonian function

$$
H(x, y, t)=\frac{1}{2} y^{2}+V(x)+p(t) G(x)-F(x, t)
$$

In order to introduce action and angle variables, we first consider the auxiliary autonomous equation:

$$
x^{\prime}=y, \quad y^{\prime}=-V^{\prime}(x)
$$

which is an integrable Hamiltonian system with Hamiltonian function

$$
H_{1}(x, y, t)=\frac{1}{2} y^{2}+V(x)
$$

The closed curves $H_{1}(x, y, t)=h>0$ are just the integral curves of (2.2).

Denote by $T(h)$ the time period of the integral curve $\Gamma_{h}$ of (2.2) defined by $H_{1}(x, y, t)=h$ and by $I$ the area enclosed by the closed curve $\Gamma_{h}$ for every $h>0$. Let $-1<-\alpha_{h}<\beta_{h}$ be such that $V\left(-\alpha_{h}\right)=V\left(\beta_{h}\right)=h$. Then by assumption (1), we have

$$
\lim _{h \rightarrow+\infty}\left(\alpha_{h}\right)=1, \quad \lim _{h \rightarrow+\infty}\left(\beta_{h}\right)=+\infty
$$

It is easy to see that

$$
I(h)=\int_{-\alpha_{h}}^{\beta_{h}} \sqrt{2(h-V(s))} d s, \quad \forall h>0 .
$$

Let us denote

$$
T_{-}(h)=\int_{-\alpha_{h}}^{0} \frac{1}{\sqrt{2(h-V(s))}} d s, \quad T_{+}(h)=\int_{0}^{\beta_{h}} \frac{1}{\sqrt{2(h-V(s))}} d s .
$$

By assumption (4), we know that the auxiliary autonomous equation is isochronous and we have the periodic $T(h)=T_{-}(h)+T_{+}(h)=2 \pi$, which yields $I(h)=2 \pi h$. Moreover, similar to the estimate in $[5,6]$, we have

$$
\left|h^{k} T_{+}^{(k)}(h)\right| \leq C \cdot \frac{1}{\sqrt{h}}, \quad k>0
$$


and

$$
\left|h^{k} T_{-}^{(k)}(h)\right| \leq C \cdot \frac{1}{\sqrt{h}}, \quad k \geq 0 .
$$

We now carry out the standard reduction to the action-angle variables. For this purpose, we define the generating function $S(x, I)=\int_{C} \sqrt{2(h-V(s))} d s$, where $C$ is the part of the closed curve $\Gamma_{h}$ connecting the point on the $y$-axis and the point $(x, y)$.

We define the well-known map $(\theta, I) \rightarrow(x, y)$ by

$$
y=\frac{\partial S}{\partial x}(x, I), \quad \theta=\frac{\partial S}{\partial I}(x, I),
$$

which is symplectic, since

$$
\begin{aligned}
& d x \wedge d y=d x \wedge\left(S_{x x} d x+S_{x I} d I\right)=S_{x I} d x \wedge d I \\
& d \theta \wedge d I=\left(S_{I x} d x+S_{I I} d I\right) \wedge d I=S_{I x} d \wedge d I .
\end{aligned}
$$

From the above discussion, we easily get

$$
\begin{aligned}
& \theta= \begin{cases}\int_{-\alpha_{h}}^{x} \frac{1}{\sqrt{2(h(x, y)-V(s))}} d s, & \text { if } y \geq 0, \\
2 \pi-\int_{-\alpha_{h}}^{x} \frac{1}{\sqrt{2(h(x, y)-V(s))}} d s, & \text { if } y<0,\end{cases} \\
& I(x, y)=\int_{-\alpha_{h}}^{\beta_{h}} \sqrt{2(h(x, y)-V(s))} d s .
\end{aligned}
$$

In the new variables $(\theta, I)$, the system (2.1) becomes

$$
\theta^{\prime}=\frac{\partial H}{\partial I}, \quad I^{\prime}=-\frac{\partial H}{\partial \theta}
$$

where

$$
H(\theta, I, t)=I+2 \pi p(t) G(x(\theta, I))-2 \pi F(x(\theta, I), t) .
$$

\subsection{New action and angle variables}

Now we are concerned with the Hamiltonian system (2.5) with the Hamiltonian function $H(\theta, I, t)$ given by $(2.6)$. Note that

$$
I d \theta-H d t=-(H d t-I d \theta) .
$$

This means that if one can solve $I$ from (2.6) as a function of $H$ ( $\theta$ and $t$ as parameters), then

$$
\frac{d H}{d \theta}=-\frac{\partial I}{\partial t}(t, H, \theta), \quad \frac{d t}{d \theta}=-\frac{\partial I}{\partial H}(t, H, \theta)
$$

is also a Hamiltonian system with Hamiltonian function $I$ and now the action, angle, and time variables are $H, t$, and $\theta$. 
Let

$$
R(\theta, I, t)=2 \pi p(t) G(x(\theta, I))-2 \pi F(x(\theta, I), t) .
$$

In order to estimate $R$, we need the estimate on the functions $x(I, \theta)$. For this purpose, we introduce the following lemma proved in $[3,6]$.

Lemma 1 There is a constant $C$ such that

$$
I^{k} \frac{\partial^{k} x}{\partial I^{k}} \leq C(1+x) \leq C \sqrt{I} \quad \text { for } 0 \leq k \leq 6 .
$$

By $\left(5^{\prime}\right),(2.8)$, and Lemma 1, we can directly get the following lemma.

Lemma 2 The following estimates hold:

$$
\left|\frac{\partial^{k+l} R(\theta, I, t)}{\partial I^{k} \partial t^{l}}\right|<I^{\frac{\alpha+1}{2}}
$$

for $k+l \leq 6$.

Using (2.6), we have

$$
\frac{\partial H}{\partial I}=1+\frac{\partial R}{\partial I} \rightarrow 1, \quad \text { as } I \rightarrow+\infty .
$$

Hence, by the implicit function theorem, there exists a function $R_{1}=R_{1}(t, H, \theta)$ such that

$$
I=H-R_{1}(t, H, \theta) .
$$

The function $R_{1}$ is defined implicitly by

$$
R_{1}(t, H, \theta)=R\left(\theta, H-R_{1}, t\right) .
$$

Now we give the estimates of $R_{1}(t, H, \theta)$. By a direct computation, we have the following.

Lemma $3\left|H^{k} \frac{\partial^{k+l} R_{1}(t, H, \theta)}{\partial H^{k} \partial t^{l}}\right|<H^{\frac{\alpha+1}{2}}$ for $k+l \leq 6$.

Proof Case $m=0$. By (2.9), Lemma 2 and noticing that $\frac{H}{I} \rightarrow 1$ as $I \rightarrow+\infty$, we have

$$
\left|R_{1}(t, H, \theta)\right|=\left|R\left(t, H-R_{1}, \theta\right)\right| \leq C \cdot\left|H-R_{1}\right|^{\frac{\alpha+1}{2}} \leq C \cdot|H|^{\frac{\alpha+1}{2}} .
$$

Case $m=1$. Taking derivatives on both sides of (2.9) with respect to $H$, we have

$$
\frac{\partial R_{1}}{\partial H}=\frac{1}{\pi} \frac{1}{1+\frac{1}{\frac{\partial R}{\partial I}} .}
$$

By Lemma 2, we have

$$
\left|\frac{\partial R}{\partial I}\right| \leq C \cdot I^{\frac{-1+\alpha}{2}} \leq C \cdot H^{\frac{-1+\alpha}{2}} .
$$


Since

$$
\left|\frac{1}{\frac{\partial R}{\partial H}}\right| \gg 1 \quad \text { as } H \rightarrow+\infty
$$

we have

$$
\left|H \frac{\partial R_{1}}{\partial H}\right| \leq C \cdot H^{\frac{1+\alpha}{2}}
$$

We suppose that

$$
\left|H^{m} \frac{\partial^{m} R_{1}(H, t, \theta)}{\partial H^{m}}\right| \leq H^{\frac{\alpha+1}{2}}
$$

holds where $m=k-1$. We will prove that (2.10) also holds when $m=k, 1 \leq k \leq 6$.

By direct calculation, we have

$$
\frac{\partial^{k} R_{1}}{\partial H^{k}}=\frac{\sum c_{n j_{1} \cdots j_{n}} \frac{\partial^{n} R}{\partial I^{n}} \frac{\partial^{j_{1}}}{\partial H^{j_{1}}}\left(H-R_{1}\right) \cdots \frac{\partial^{j_{n}}}{\partial H^{j_{n}}}\left(H-R_{1}\right)}{1+\frac{\partial R}{\partial I}},
$$

where $1 \leq n \leq k, j_{1}+\cdots+j_{n}=k, 1 \leq j_{1}, \ldots, j_{n}<k$.

By (2.10), we have

$$
\left|\frac{\partial^{\sigma}\left(H-R_{1}\right)}{\partial H^{\sigma}}\right| \leq C \cdot\left|\frac{\partial\left(H-R_{1}\right)}{\partial H}\right|^{\sigma}
$$

where $1<\sigma \leq k-1$.

By (2.11), (2.12), and Lemma 2, we have

$$
\left|\frac{\partial^{k} R_{1}}{\partial H^{k}}\right| \leq C \cdot\left|\frac{\partial^{k} R}{\partial I^{k}}\left(\frac{\partial\left(H-R_{1}\right)}{\partial H}\right)^{k}\right| \leq C \cdot\left|\frac{\partial^{k} R}{\partial I^{k}}\right| \leq C \cdot H^{\frac{\alpha+1}{2}-k}
$$

then we proved that (2.10) holds when $m=k$. Thus we proved Lemma 3.

Set

$$
\begin{aligned}
R_{2}(t, H, \theta) & =R(\theta, H, t)-R_{1}(t, H, \theta) \\
& =-\int_{0}^{1} \frac{\partial R}{\partial I}\left(\theta, H-s R_{1}, t\right) R_{1} d s .
\end{aligned}
$$

By similarity to the proofs of Lemmas 2 and 3, we have the following.

Lemma 4 The following estimates hold:

$$
\left|\frac{\partial^{k+l} R_{2}(t, H, \theta)}{\partial H^{k} \partial t^{l}}\right|<H^{\alpha}
$$

for $k+l \leq 6$ 
Remark 1 By the proofs and estimates of $R, R_{1}, R_{2}$, we can easily see that $R_{1}$ is the main (twist) term of $R$ and $R_{2}$ is the small term of $R$.

Now the new Hamiltonian function $I=I(t, H, \theta)$ is written in the form

$$
\begin{aligned}
I & =H-R_{1}(\theta, H, t)+R_{2}(t, H, \theta) \\
& =H-2 \pi p(t) G(x(\theta, H))+2 \pi F(x(\theta, H), t)+R_{2} .
\end{aligned}
$$

The system (2.7) is of the form

$$
\left\{\begin{array}{l}
\frac{d t}{d \theta}=\frac{\partial I}{\partial H}=1-2 \pi \frac{\partial x}{\partial H}(\theta, H)\left(g(x(\theta, H)) p(t)-F_{x}(x(\theta, H), t)\right)+\frac{\partial R_{2}}{\partial H}(t, H, \theta), \\
\frac{d H}{d \theta}=-\frac{\partial I}{\partial t}=2 \pi p^{\prime}(t) G(x(\theta, H))-2 \pi F_{t}(x(\theta, I), t)-\frac{\partial R_{2}}{\partial t}(t, H, \theta) .
\end{array}\right.
$$

Introduce a new action variable $\rho \in[1,2]$ and a parameter $\epsilon>0$ by $H=\epsilon^{-2} \rho$. Then $H \gg$ $1 \Leftrightarrow 0<\epsilon \ll 1$. Under this transformation, the system (2.13) is changed into the form

$$
\left\{\begin{array}{l}
\frac{d t}{d \theta}=1-2 \pi \frac{\partial x}{\partial H}\left(\theta, \epsilon^{-2} \rho\right)\left(g\left(\theta, \epsilon^{-2} \rho\right) p(t)-F_{x}\left(x\left(\theta, \epsilon^{-2} \rho\right), t\right)\right)+\frac{\partial R_{2}}{\partial H}\left(t, \epsilon^{-2} \rho, \theta\right), \\
\frac{d \rho}{d \theta}=2 \pi \epsilon^{2} p^{\prime}(t) G\left(x\left(\theta, \epsilon^{-2} \rho\right)\right)-2 \pi \epsilon^{2} F_{t}(x(\theta, I), t)-\epsilon^{2} \frac{\partial R_{2}}{\partial t}\left(t, \epsilon^{-2} \rho, \theta\right),
\end{array}\right.
$$

which is also a Hamiltonian system, with the new Hamiltonian function

$$
\Gamma(t, \rho, \theta ; \epsilon)=\rho-2 \pi \epsilon^{-2} p(t) G\left(x\left(\theta, \epsilon^{-2} \rho\right)\right)+2 \pi \epsilon^{-2} F\left(x\left(\theta, \epsilon^{-2} \rho\right), t\right)+\epsilon^{-2} \frac{\partial R_{2}}{\partial t}\left(t, \epsilon^{-2} \rho, \theta\right) .
$$

Obviously, if $\epsilon \ll 1$, the solution $\left(t\left(\theta, t_{0}, \rho_{0}\right), \rho\left(\theta, t_{0}, \rho_{0}\right)\right)$ of $(2.14)$ with the initial date $\left(t_{0}, \rho_{0}\right) \in R \times[1,2]$ is defined in the interval $\theta \in[0,2 \pi]$ and $\rho\left(\theta, t_{0}, \rho_{0}\right) \in\left[\frac{1}{2}, 3\right]$. So the Poincaré map of (2.14) is well defined in the domain $R \times[1,2]$.

Lemma 5 ([6, Lemma 5.1]) The Poincaré map of (2.14) has the intersection property.

The proof is similar to the corresponding one in [6].

For convenience we introduce the notation $O_{k}(1)$ and $o_{k}(1)$. We say a function $f(t, \rho$, $\theta, \epsilon) \in O_{k}(1)$ if $f$ is smooth in $(t, \rho)$ and for $k_{1}+k_{2} \leq k$,

$$
\left|\frac{\partial^{k_{1}+k_{2}}}{\partial t^{k_{1}} \partial \rho^{k_{2}}} f(t, \rho, \theta, \epsilon)\right| \leq C,
$$

for some constant $C>0$ which is independent of the arguments $t, \rho, \theta, \epsilon$.

Similarly, we say $f(t, \rho, \theta, \epsilon) \in o_{k}(1)$ if $f$ is smooth in $(t, \rho)$ and for $k_{1}+k_{2} \leq k$,

$$
\lim _{\epsilon \rightarrow 0}\left|\frac{\partial^{k_{1}+k_{2}}}{\partial t^{k_{1}} \partial \rho^{k_{2}}} f(t, \rho, \theta, \epsilon)\right|=0 \text {, }
$$

uniformly in $(t, \rho, \theta)$.

\subsection{Poincaré map and twist theorems}

We will use Ortega's small twist theorem to prove that the Poincaré map $P$ has an invariant closed curve, if $\epsilon$ is sufficiently small. The earlier result was due to Moser [11-13]. Later, Orgeta [10] improved the previous results. Let us first recall the theorem in [10]. 
Lemma 6 (Ortega's Theorem) Let $A=\mathbb{S}^{1} \times[a, b]$ be a finite cylinder with universal cover $\mathbb{A}=\mathbb{R} \times[a, b]$. The coordinate in $\mathbb{A}$ is denoted by $(\tau, v)$. Consider the map

$$
\bar{f}: A \rightarrow \mathbb{S} \times \mathbb{R}
$$

We assume that the map has the intersection property. Suppose that $f: A \rightarrow \mathbb{R} \times \mathbb{R}$, $\left(\tau_{0}, v_{0}\right) \rightarrow\left(\tau_{1}, v_{1}\right)$ is a lift of $\bar{f}$ and it has the form

$$
\left\{\begin{array}{l}
\tau_{1}=\tau_{0}+2 N \pi+\delta l_{1}\left(\tau_{0}, \nu_{0}\right)+\delta \tilde{g}_{1}\left(\tau_{0}, \nu_{0}\right), \\
v_{1}=v_{0}+\delta l_{2}\left(\tau_{0}, \nu_{0}\right)+\delta \tilde{g}_{2}\left(\tau_{0}, v_{0}\right)
\end{array}\right.
$$

where $N$ is an integer, $\delta \in(0,1)$ is a parameter. The functions $l_{1}, l_{2}, \tilde{g}_{1}$, and $\tilde{g}_{2}$ satisfy

$$
\begin{aligned}
& l_{1} \in C^{6}(A), \quad l_{1}\left(\tau_{0}, v_{0}\right)>0, \quad \frac{\partial l_{1}}{\partial v_{0}}\left(\tau_{0}, v_{0}\right)>0, \quad \forall\left(\tau_{0}, v_{0}\right) \in A, \\
& l_{2}(\cdot, \cdot), \tilde{g}_{1}(\cdot, \cdot, \epsilon), \tilde{g}_{2}(\cdot, \cdot, \epsilon) \in C^{5}(A) .
\end{aligned}
$$

In addition, we assume that there is a function $I: A \rightarrow R$ satisfying

$$
I \in C^{6}(A), \quad \frac{\partial I}{\partial v_{0}}\left(\tau_{0}, v_{0}\right)>0, \quad \forall\left(\tau_{0}, v_{0}\right) \in A
$$

and

$$
l_{1}\left(\tau_{0}, v_{0}\right) \cdot \frac{\partial I}{\partial \tau_{0}}\left(\tau_{0}, v_{0}\right)+l_{2}\left(\tau_{0}, v_{0}\right) \cdot \frac{\partial I}{\partial \nu_{0}}\left(\tau_{0}, v_{0}\right)=0, \quad \forall\left(\tau_{0}, v_{0}\right) \in A .
$$

Moreover, suppose that there are two numbers $\tilde{a}$ and $\tilde{b}$ such that $a<\tilde{a}<\tilde{b}<b$ and

$$
I_{M}(a)<I_{m}(\tilde{a}) \leq I_{M}(\tilde{a})<I_{m}(\tilde{b}) \leq I_{M}(\tilde{b})<I_{m}(b)
$$

where

$$
I_{M}(r)=\max _{\rho \in S^{1}} I\left(\rho_{0}, \tau_{0}\right), \quad I_{m}(r)=\min _{\rho \in S^{1}} I\left(\rho_{0}, \tau_{0}\right) .
$$

Then there exist $\epsilon>0$ and $\Delta>0$ such that, if $\delta<\Delta$ and

$$
\left\|\tilde{g}_{1}(\cdot, \cdot, \epsilon)\right\|_{C^{5}(A)}+\left\|\tilde{g}_{2}(\cdot, \cdot, \epsilon)\right\|_{C^{5}(A)}<\epsilon,
$$

the mapping $\bar{f}$ has an invariant curve in $\Gamma_{A}$. The constant $\epsilon$ is independent of $\delta$.

We make the ansatz that the solution of (2.14) with the initial condition $(t(0), \rho(0))=$ $\left(t_{0}, \rho_{0}\right)$ is of the form

$$
t=t_{0}+\theta+\epsilon^{1-\alpha} \Sigma_{1}\left(t_{0}, \rho_{0}, \theta ; \epsilon\right), \quad \rho=\rho_{0}+\epsilon^{1-\alpha} \Sigma_{2}\left(t_{0}, \rho_{0}, \theta ; \epsilon\right) .
$$

Then the Poincaré map of (2.14) is

$$
P: t_{1}=t_{0}+2 \pi+\epsilon^{1-\alpha} \Sigma_{1}\left(t_{0}, \rho_{0}, 2 \pi ; \epsilon\right), \quad \rho_{1}=\rho_{0}+\epsilon^{1-\alpha} \Sigma_{2}\left(t_{0}, \rho_{0}, 2 \pi ; \epsilon\right) .
$$


The functions $\Sigma_{1}$ and $\Sigma_{2}$ satisfy

$$
\left\{\begin{aligned}
\Sigma_{1}= & 2 \pi \epsilon^{\alpha-1} \int_{0}^{\theta} \frac{\partial x}{\partial H}\left(\theta, \epsilon^{-2} \rho\right)\left(g(x) p(t)-F_{x}\left(x\left(\theta, \epsilon^{-2} \rho\right), t\right)\right) d \theta \\
& +\epsilon^{\alpha-1} \int_{0}^{\theta} \frac{\partial R_{2}}{\partial H}\left(\theta, \epsilon^{-2} \rho\right) d \theta, \\
\Sigma_{2}= & 2 \pi \epsilon^{\alpha+1} \int_{0}^{\theta} G\left(x\left(\theta, \epsilon^{-2} \rho\right)\right) p^{\prime}(t)-F_{t}\left(x\left(\theta, \epsilon^{-2} \rho\right), t\right) d \theta \\
& -\epsilon^{\alpha+1} \int_{0}^{\theta} \frac{\partial R_{2}}{\partial t}\left(\theta, \epsilon^{-2} \rho\right) d \theta,
\end{aligned}\right.
$$

where $t=t_{0}+\theta+\epsilon^{1-\alpha} \Sigma_{1}, \rho=\rho_{0}+\epsilon^{1-\alpha} \Sigma_{2}$. By Lemmas 1 and 3, we know that

$$
\left|\Sigma_{1}\right|+\left|\Sigma_{2}\right| \leq C \quad \text { for } \theta \in[0,2 \pi]
$$

Moreover we can prove that

$$
\Sigma_{1}, \Sigma_{2} \in O_{6}(1)
$$

Lemma 7 The following estimates hold:

$$
\begin{aligned}
& G\left(x\left(\theta, \epsilon^{-2} \rho\right)\right)-G\left(x\left(\theta, \epsilon^{-2} \rho_{0}\right)\right) \in \epsilon^{-2 \alpha} O_{6}(1), \\
& g\left(x\left(\theta, \epsilon^{-2} \rho\right)\right) \frac{\partial x}{\partial H}-g\left(x\left(\theta, \epsilon^{-2} \rho_{0}\right)\right) \frac{\partial x}{\partial H} \in \epsilon^{2-2 \alpha} O_{6}(1) .
\end{aligned}
$$

Proof We only consider $x \geq 0$, since the case $x \leq 0$ can be proved similarly.

Let

$$
\begin{aligned}
\Delta\left(t--_{0}, \rho_{0}, \theta\right)= & G\left(x\left(\theta, \epsilon^{-2} \rho\right)\right)-G\left(x\left(\theta, \epsilon^{-2} \rho_{0}\right)\right) \\
= & \int_{0}^{1} g\left(x\left(\theta, \epsilon^{-2} \rho_{0}+s \epsilon^{-1-\alpha} \Sigma_{2}\right)\right) \\
& \cdot \frac{\partial x}{\partial H}\left(\theta, \epsilon^{-2} \rho_{0}+s \epsilon^{-1-\alpha} \Sigma_{2}\right) \epsilon^{-1-\alpha} \Sigma_{2} d s .
\end{aligned}
$$

By $\left(6^{\prime}\right)$, Lemma 2 and (2.21), we have

$$
\begin{aligned}
\left|\Delta\left(t--_{0}, \rho_{0}, \theta\right)\right| & \leq C \cdot\left(\epsilon^{-2} \rho_{0}+s \epsilon^{-1-\alpha} \Sigma_{2}\right)^{\frac{\alpha}{2}}\left(\epsilon^{-2} \rho_{0}+s \epsilon^{-1-\alpha} \Sigma_{2}\right)^{-\frac{1}{2}} \epsilon^{-1-\alpha} \Sigma_{2} \\
& \leq C \cdot\left(\epsilon^{-2} \rho_{0}+s \epsilon^{-1-\alpha} \Sigma_{2}\right)^{\frac{\alpha-1}{2}} \epsilon^{-1-\alpha} \\
& \leq C \cdot \epsilon^{-2 \alpha} .
\end{aligned}
$$

Taking the derivative with respect to $\rho_{0}$ in both sides of $\Delta\left(t---0, \rho_{0}, \theta\right)$, we have

$$
\begin{aligned}
\frac{\partial \Delta}{\partial \rho_{0}}= & \int_{0}^{1}\left[g^{\prime}(x) \frac{\partial x}{\partial H} \frac{1+s \epsilon^{1-\alpha} \frac{\partial \Sigma_{2}}{\partial \rho_{0}}}{\epsilon^{2}} \frac{\partial x}{\partial H} \epsilon^{-1-\alpha} \Sigma_{2}+g(x) \frac{\partial^{2} x}{\partial H^{2}} \frac{1+s \epsilon \frac{\partial \Sigma_{2}}{\partial \rho_{0}}}{\epsilon^{2}} \epsilon^{-1-\alpha} \Sigma_{2}\right. \\
& \left.+g(x) \frac{\partial x}{\partial H} \frac{\partial \Sigma_{2}}{\partial \rho_{0}} \epsilon^{-1-\alpha}\right] d s .
\end{aligned}
$$

Similar to the estimates of $|\Delta|$ and noticing $|\Delta| \leq C \cdot \epsilon^{-2 \alpha}$, we have

$$
\left|\frac{\partial \Delta}{\partial \rho_{0}}\right| \leq C \cdot \epsilon^{-2 \alpha} .
$$


Now we will estimate $\frac{\partial^{n} \Delta}{\partial \rho^{n}}, n \geq 2$. By direct calculation, we have

$$
\frac{\partial^{n} \Delta}{\partial \rho^{n}}=\int_{0}^{1} \Sigma \frac{\partial^{\Sigma j_{k 1}} g(x)}{\partial \rho^{\Sigma j_{k 1}}} \cdot \frac{\partial^{\Sigma j_{k 2}} x_{H}}{\partial \rho^{\Sigma j_{k 2}}} \cdot \frac{\partial^{\Sigma j_{k 3}} \Phi}{\partial \rho^{\Sigma j_{k 3}}} d s
$$

where $\Phi=\epsilon^{-1-\alpha} \Sigma_{2}, \Sigma j_{k l}=1, n \geq 2, k=1,2, \ldots, n, l=1,2,3$. For estimating $\frac{\partial^{n} \Delta}{\partial \rho^{n}}$, we need the estimates of $x_{H}$ and $g(x)$.

We firstly give the estimates of $x_{H}$. By direct calculation, we get

$$
\frac{\partial^{n} x_{H}}{\partial \rho^{n}}=\Sigma \frac{\partial^{k} g(x)}{\partial H^{k}} \cdot \frac{\partial^{i_{1}} \Psi}{\partial \rho^{i_{1}}} \cdots \frac{\partial^{i_{k}} \Psi}{\partial \rho^{i_{k}}},
$$

where $\Psi=\epsilon^{-2}+s \epsilon^{-1-\alpha} \frac{\partial\left(\Sigma_{2}\right)}{\partial \rho}, n \geq 1, k+i_{1}+\cdots+i_{k}=n$.

Since $\Psi=\epsilon^{-2}+s \epsilon^{-1-\alpha} \frac{\partial\left(\Sigma_{2}\right)}{\partial \rho}$, we have

$$
\Psi \leq C \cdot \epsilon^{-2}, \quad \frac{\partial^{\tau} \Psi}{\partial \rho^{\tau}} \leq C \cdot\left(\frac{\partial \Psi}{\partial \rho}\right)^{\tau}, \quad \tau>1
$$

By (2.25) and (2.26), we have

$$
\frac{\partial^{n} x_{H}}{\partial \rho^{n}} \leq C \cdot \frac{\partial^{n} g(x)}{\partial H^{n}}(\Psi)^{n} \leq C \cdot \epsilon^{1+2 n} \cdot\left(\epsilon^{-2}\right)^{n}=C \cdot \epsilon .
$$

We now give the estimates of $g(x)$. By $\left(6^{\prime}\right),(2.26),(2.27)$, and Lemma 1, we have

$$
g(x) \leq C \cdot \epsilon^{-\alpha}, \quad \frac{\partial g(x)}{\partial \rho}=g^{\prime}(x) x_{H} \Psi \leq C \cdot \epsilon^{1-\alpha} \cdot \epsilon \cdot \epsilon^{-\alpha} \leq C \cdot \epsilon^{-\alpha} .
$$

We suppose that the following inequality holds:

$$
\frac{\partial^{n-1} g(x)}{\partial \rho^{n-1}} \leq C \cdot \epsilon^{-\alpha}, \quad n \geq 1
$$

By direct calculation, we have

$$
\frac{\partial^{n} g(x)}{\partial \rho^{n}}=\Sigma \frac{\partial^{j_{1}} g(x)}{\partial \rho^{j_{1}}} \cdot \frac{\partial^{j_{2}} x_{H}}{\partial \rho^{j_{2}}} \cdot \frac{\partial^{j_{3}} \Psi}{\partial \rho^{j_{3}}},
$$

where $j_{i} \geq 0, j_{1}+j_{2}+j_{3}=n-1$. By the estimates of $\Psi(2.26)$, the estimates of $x_{H}(2.27)$ and (2.28), (2.29), we have

$$
\frac{\partial^{n} g(x)}{\partial \rho^{n}} \leq C \cdot \epsilon^{1-\alpha} \cdot \epsilon \cdot \epsilon^{-2}=C \cdot \epsilon^{-\alpha} .
$$

Since we have the estimates of $\Psi, x_{H}$, and $g(x)$, now we can give the estimates of $\frac{\partial^{n} \Delta}{\partial \rho^{n}}$. By (2.26), (2.27), (2.30), and (2.24), we have the estimate of $\Delta$ :

$$
\begin{aligned}
\frac{\partial^{n} \Delta}{\partial \rho^{n}} & =\int_{0}^{1} \Sigma \frac{\partial^{\Sigma j_{k 1}} g(x)}{\partial \rho^{\Sigma j_{k 1}}} \cdot \frac{\partial^{\Sigma j_{k 2}} x_{H}}{\partial \rho^{\Sigma j_{k 2}}} \cdot \frac{\partial^{\Sigma j_{k 3}} \Phi}{\partial \rho^{\Sigma j_{k 3}}} d s, \\
& \leq C \cdot \epsilon^{-\alpha} \cdot \epsilon \cdot \epsilon^{-1-\alpha} \\
& =\epsilon^{-2 \alpha}
\end{aligned}
$$


which means that

$$
G\left(x\left(\theta, \epsilon^{-2} \rho\right)\right)-G\left(x\left(\theta, \epsilon^{-2} \rho_{0}\right)\right) \in \epsilon^{-2 \alpha} O_{6}(1) .
$$

The estimates for $\frac{\partial x}{\partial H}\left(\theta, \epsilon^{-2} \rho\right) g\left(x\left(\theta, \epsilon^{-2} \rho\right)\right)-\frac{\partial x}{\partial H}\left(\theta, \epsilon^{-2} \rho_{0}\right) g\left(x\left(\theta, \epsilon^{-2} \rho_{0}\right)\right)$ follow from a similar argument, and we omit it here. Thus Lemma 7 is proved.

Now we turn to giving an asymptotic expression of the Poincare map of (2.13), that is, we study the behavior of the functions $\Sigma_{1}$ and $\Sigma_{2}$ at $\theta=2 \pi$ as $\epsilon \rightarrow 0$.

In order to estimate $\Sigma_{1}$ and $\Sigma_{2}$, we introduce the following definition and lemma. Let $\Theta_{+}(I)$ be the subset of the interval $[0,2 \pi]$ such that $\theta \in \Theta_{+}(I), x(\theta, I)>0$ and $\Theta_{-}(I)$ satisfies $\theta \in \Theta_{-}(I), x(\theta, I)<0$. By (2.3) and (2.4), similar to the computation in [6], there is a function $\eta \in \epsilon O_{6}(1)$ such that $T_{-}(h)=T_{-}\left(\frac{I}{2 \pi}\right)=2 \eta\left(t_{0}, \rho_{0}, \theta_{0} ; \epsilon\right)$. By the definition of $\Theta_{+}$and $\Theta_{-}$, we have

$$
\text { measure } \Theta_{-}=2 \eta, \quad \text { measure } \Theta_{+}=2 \pi-2 \eta \text {. }
$$

Moreover we will use the following lemma.

Lemma 8 ([6, Lemma 4.1]) For $\theta \in \Theta_{+}(I)$, the function $x$ has the following expression:

$$
x(\theta, I)=2 \sqrt{\frac{I}{\pi}} \sin \left(\frac{\theta}{2}-\frac{T_{-}(h)}{4}\right)+\sqrt{\frac{I}{\pi}} X\left(\theta-\frac{T_{-}(h)}{4}, I\right),
$$

and

$$
\lim _{I \rightarrow+\infty} \sum_{k=1}^{6}\left|I^{k-\frac{1}{2}}\left(\frac{\partial^{k}}{\partial I^{k}}\left(x(\theta, I)-2 \sqrt{\frac{I}{\pi}} \sin \left(\frac{\theta}{2}-\frac{T_{-}(h)}{4}\right)\right)\right)\right|=0 .
$$

Now we can give the estimates of $\Sigma_{1}$ and $\Sigma_{2}$.

\section{Lemma 9}

$$
\begin{aligned}
& \Sigma_{1}\left(t_{0}, \rho_{0}, 2 \pi ; \epsilon\right)=-2^{\alpha+1} \pi^{\frac{1}{2}} \rho_{0}^{\frac{\alpha-1}{2}} \int_{0}^{2 \pi} \sin \frac{\theta}{2}\left|\sin \frac{\theta}{2}\right|^{\alpha} p\left(t_{0}+\theta\right) d \theta+o_{6}(1), \\
& \Sigma_{2}\left(t_{0}, \rho_{0}, 2 \pi ; \epsilon\right)=-2^{2+\alpha} \pi^{\frac{1}{2}} \rho_{0}^{\frac{\alpha+1}{2}} \int_{0}^{2 \pi} \sin \frac{\theta}{2}\left|\sin \frac{\theta}{2}\right|^{\alpha} p^{\prime}\left(t_{0}+\theta\right) d \theta+o_{6}(1) .
\end{aligned}
$$

Proof Firstly we consider $\Sigma_{1}$. By Lemmas 4, 7, and (2.21), we have

$$
\begin{aligned}
\Sigma_{1}\left(t_{0}, \rho_{0}, 2 \pi ; \epsilon\right) \\
=-2 \pi \epsilon^{\alpha-1} \int_{0}^{2 \pi} \frac{\partial x}{\partial H}\left(\theta, \epsilon^{-2} \rho\right)\left(g\left(x\left(\theta, \epsilon^{-2} \rho\right)\right) p(t)-F_{x}\left(x\left(\theta, \epsilon^{-2} \rho\right), t\right)\right) d \theta \\
\quad+\epsilon^{\alpha-1} \int_{0}^{2 \pi} \frac{\partial R_{2}}{\partial H}\left(\theta, \epsilon^{-2} \rho\right) d \theta \\
=-2 \pi \epsilon^{\alpha-1} \int_{0}^{2 \pi} \frac{\partial x}{\partial H}\left(\theta, \epsilon^{-2} \rho_{0}\right)\left(g\left(x\left(\theta, \epsilon^{-2} \rho\right)\right) p\left(t_{0}+\theta\right)-F_{x}\left(x\left(\theta, \epsilon^{-2} \rho_{0}\right), t\right)\right) d \theta
\end{aligned}
$$




$$
\begin{aligned}
& +\epsilon^{1-\alpha} O_{6}(1) \\
= & -2 \pi \epsilon^{\alpha-1} \int_{\Theta_{+}} \frac{\partial x}{\partial H}\left(\theta, \epsilon^{-2} \rho_{0}\right)\left(g\left(x\left(\theta, \epsilon^{-2} \rho\right)\right) p\left(t_{0}+\theta\right)-F_{x}\left(x\left(\theta, \epsilon^{-2} \rho_{0}\right), t\right)\right) d \theta \\
& -2 \pi \epsilon^{\alpha-1} \int_{\Theta_{-}} \frac{\partial x}{\partial H}\left(\theta, \epsilon^{-2} \rho_{0}\right)\left(g\left(x\left(\theta, \epsilon^{-2} \rho\right)\right) p\left(t_{0}+\theta\right)-F_{x}\left(x\left(\theta, \epsilon^{-2} \rho_{0}\right), t\right)\right) d \theta \\
& +\epsilon^{1-\alpha} O_{6}(1) .
\end{aligned}
$$

By Lemma 1, we know that when $x \in \Theta_{-}$

$$
x\left(\left(\theta, \epsilon^{-2} \rho_{0}\right)\right) \in O_{6}(1), \quad \frac{\partial x}{\partial H}\left(\theta, \epsilon^{-2} \rho_{0}\right) \in \epsilon^{2} O_{6}(1) .
$$

By $(2.32)$ and $\left(6^{\prime}\right)$, we have

$$
\begin{aligned}
\Sigma_{1}\left(t_{0}, \rho_{0}, 2 \pi ; \epsilon\right)= & -2 \pi \epsilon^{\alpha-1} \int_{\Theta_{+}} \frac{\partial x}{\partial H}\left(\theta, \epsilon^{-2} \rho_{0}\right)\left(|x|^{\alpha} p\left(t_{0}+\theta\right)-F_{x}\left(x\left(\theta, \epsilon^{-2} \rho_{0}\right), t\right)\right) d \theta \\
& +\epsilon^{1-\alpha} O_{6}(1) .
\end{aligned}
$$

By Lemma 8, we have

$$
\begin{aligned}
\Sigma_{1}\left(t_{0}, \rho_{0}, 2 \pi ; \epsilon\right)= & -2 \pi \epsilon^{\alpha-1} \int_{\Theta_{+}}\left[\frac{\epsilon}{\sqrt{\rho_{0} \pi}} \sin \left(\frac{\theta}{2}-\frac{\eta}{4}\right)\right] \\
& \cdot\left[2\left(\epsilon^{-1} \sqrt{\frac{\rho_{0}}{\pi}} \sin \left(\frac{\theta}{2}-\frac{\eta}{4}\right)\right)\right]^{\alpha} p\left(t_{0}+\theta\right) d \theta+\epsilon^{1-\alpha} O_{6}(1) \\
= & -2^{\alpha+1} \pi^{\frac{1-\alpha}{2}} \rho_{0}^{\frac{\alpha-1}{2}} \int_{\Theta_{+}} \sin \frac{\theta}{2}\left|\sin \frac{\theta}{2}\right|^{\alpha} p\left(t_{0}+\theta\right) d \theta+o_{6}(1) \\
= & -2^{\alpha+1} \pi^{\frac{1-\alpha}{2}} \rho_{0}^{\frac{\alpha-1}{2}} \int_{0}^{2 \pi} \sin \frac{\theta}{2}\left|\sin \frac{\theta}{2}\right|^{\alpha} p\left(t_{0}+\theta\right) d \theta+o_{6}(1) .
\end{aligned}
$$

Now we consider $\Sigma_{2}$ :

$$
\begin{aligned}
\Sigma_{2}= & 2 \pi \epsilon^{\alpha+1} \int_{0}^{2 \pi} G\left(x\left(\theta, \epsilon^{-2} \rho\right)\right) p^{\prime}(t) d \theta-2 \pi \epsilon^{\alpha+1} \int_{0}^{2 \pi} F_{t}\left(x\left(\theta, \epsilon^{-2} \rho\right), t\right) d \theta \\
& -\epsilon^{\alpha+1} \int_{0}^{2 \pi} \frac{\partial R_{2}}{\partial t}\left(\theta, \epsilon^{-2} \rho\right) d \theta .
\end{aligned}
$$

By $\left(6^{\prime}\right)$, Lemmas 4 and 7 , and noticing that

$$
\lim _{x \rightarrow+\infty} \frac{F(x, t)}{|x|^{\alpha+1}}=0,
$$

we have

$$
\begin{aligned}
\Sigma_{2}= & 2 \pi \epsilon^{\alpha+1} \int_{0}^{2 \pi}\left|x\left(\theta, \epsilon^{-2} \rho_{0}\right)\right|^{\alpha} x\left(\theta, \epsilon^{-2} \rho_{0}\right) p^{\prime}\left(t_{0}+\theta\right)+o_{6}(1) \\
= & 2 \pi \epsilon^{\alpha+1} \int_{\Theta_{+}}\left|x\left(\theta, \epsilon^{-2} \rho_{0}\right)\right|^{\alpha} x\left(\theta, \epsilon^{-2} \rho_{0}\right) p^{\prime}\left(t_{0}+\theta\right) \\
& +2 \pi \epsilon^{\alpha+1} \int_{\Theta_{-}}\left|x\left(\theta, \epsilon^{-2} \rho_{0}\right)\right|^{\alpha} x\left(\theta, \epsilon^{-2} \rho_{0}\right) p^{\prime}\left(t_{0}+\theta\right)+o_{6}(1) .
\end{aligned}
$$


By Lemmas 1,8 , and (2.32)

$$
\begin{aligned}
\Sigma_{2}= & 2 \pi \epsilon^{\alpha+1} \int_{\Theta_{+}}\left|x\left(\theta, \epsilon^{-2} \rho_{0}\right)\right|^{\alpha} x\left(\theta, \epsilon^{-2} \rho_{0}\right) p^{\prime}\left(t_{0}+\theta\right)+o_{6}(1) \\
= & 2 \pi \epsilon^{\alpha+1} \int_{\Theta_{+}}\left(2 \epsilon^{-1} \sqrt{\frac{\rho_{0}}{\pi}} \sin \left(\frac{\theta}{2}-\frac{\eta}{4}\right)\right) \\
& \cdot\left|2 \epsilon^{-1} \sqrt{\frac{\rho_{0}}{\pi}} \sin \left(\frac{\theta}{2}-\frac{\eta}{4}\right)\right|^{\alpha} \cdot p^{\prime}(t) d \theta+o_{6}(1) \\
= & -2^{2+\alpha} \pi^{\frac{1-\alpha}{2}} \rho_{0}^{\frac{\alpha+1}{2}} \int_{\Theta_{+}} \sin \frac{\theta}{2}\left|\sin \frac{\theta}{2}\right|^{\alpha} p^{\prime}\left(t_{0}+\theta\right) d \theta+o_{6}(1) \\
= & -2^{2+\alpha} \pi^{\frac{1-\alpha}{2}} \rho_{0}^{\frac{\alpha+1}{2}} \int_{0}^{2 \pi} \sin \frac{\theta}{2}\left|\sin \frac{\theta}{2}\right|^{\alpha} p^{\prime}\left(t_{0}+\theta\right) d \theta+o_{6}(1) .
\end{aligned}
$$

Let

$$
\begin{aligned}
& \Psi_{1}\left(t_{0}, \rho_{0}\right)=-2^{\alpha+1} \pi^{\frac{1-\alpha}{2}} \rho_{0}^{\frac{\alpha-1}{2}} \int_{0}^{2 \pi} \sin \frac{\theta}{2}\left|\sin \frac{\theta}{2}\right|^{\alpha} p\left(t_{0}+\theta\right) d \theta \\
& \Psi_{2}\left(t_{0}, \rho_{0}\right)=-2^{2+\alpha} \pi^{\frac{1-\alpha}{2}} \rho_{0}^{\frac{\alpha+1}{2}} \int_{0}^{2 \pi} \sin \frac{\theta}{2}\left|\sin \frac{\theta}{2}\right|^{\alpha} p^{\prime}\left(t_{0}+\theta\right) d \theta .
\end{aligned}
$$

Then there are two functions $\phi_{1}$ and $\phi_{2}$ such that the Poincaré map of (2.14), given by (2.20), is of the form

$$
P: \quad t_{1}=t_{0}+2 \pi+\epsilon^{1-\alpha} \Psi_{1}\left(t_{0}, \rho_{0}\right)+\epsilon^{1-\alpha} \phi_{1}, \quad \rho_{1}=\rho_{0}+\epsilon^{1-\alpha} \Psi_{2}\left(t_{0}, \rho_{0}\right)+\epsilon^{1-\alpha} \phi_{2},
$$

where $\phi_{1}, \phi_{2} \in o_{6}(1)$.

Note that $p(t)>0$,

$$
\Psi_{1}<0, \quad \frac{\partial \Psi_{1}}{\partial \rho_{0}}>0 .
$$

Let

$$
L=\frac{\rho_{0}^{-\frac{\alpha+1}{2}}}{\int_{0}^{2 \pi} \sin \frac{\theta}{2}\left|\sin \frac{\theta}{2}\right|^{\alpha} p\left(t_{0}+\theta\right) d \theta} .
$$

Then

$$
\frac{\partial L}{\partial t_{0}} \Psi_{1}\left(t_{0}, \rho_{0}\right)+\frac{\partial L}{\partial \rho_{0}} \Psi_{2}\left(t_{0}, \rho_{0}\right)=0 .
$$

The other assumptions of Ortega's theorem are easily verified. Hence, there is an invariant curve of $P$ in the annulus $\left(t_{0}, \rho_{0}\right) \in S^{1} \times[1.2]$, which implies the boundedness of our original equation (1.5). Then Theorem 1 is proved. 
Authors' contributions

The article is a joint work of two authors who contributed equally to the final version of the paper. All authors read and approved the final manuscript.

\section{Author details}

${ }^{1}$ Key Lab of Broadband Wireless Communication and Sensor Network Technology of Ministry of Education, College of Internet of Things, Nanjing University of Posts and Telecommunications, Nanjing, 210003, People's Republic of China.

${ }^{2}$ College of Sciences, Nanjing University of Technology, Nanjing, 210009, People's Republic of China.

\section{Acknowledgements}

Thanks are given to referees whose comments and suggestions were very helpful for revising our paper. This work is supported by National Natural Science Foundation of China (Grant Nos. 60973140, 61170276, 61373135, 60873231), Major Natural Foundation of the Higher Education Institutions of Jiangsu Province, China (Grant No. 12KJA520003), the Innovation Project for postgraduate cultivation of Jiangsu Province Grant No. CXLX11-0415, the research project of Jiangsu Province Grant No. BY2013011, the Natural Science Foundation of Jiangsu Province Grant No. BK20130096.

Received: 13 September 2013 Accepted: 14 January 2014 Published: 04 Feb 2014

\section{References}

1. Littlewood, JE: Unbounded solutions of $y^{\prime \prime}+g(y)=p(t)$. J. Lond. Math. Soc. 41, 491-496 (1966)

2. Morris, GR: A case of boundedness of Littlewood's problem on oscillatory differential equations. Bull. Aust. Math. Soc. 14, 71-93 (1976)

3. Levi, M: Quasiperiodic motions in superquadratic time-periodic potential. Commun. Math. Phys. 144, 43-82 (1991)

4. Liu, B: Boundedness in asymmetric oscillations. J. Math. Anal. Appl. 231, 355-373 (1999)

5. Bonheure, D, Fabry, C, Smets, D: Periodic solutions of forced isochronous oscillators at resonance. Discrete Contin. Dyn. Syst. 8(4), 907-930 (2002)

6. Liu, B: Quasi-periodic solutions of forced isochronous oscillators at resonance. J. Differ. Equ. 246, $3471-3495$ (2009)

7. Alonso, JM, Ortega, R: Unbounded solutions of semilinear equations at resonance. Nonlinearity 9, 1099-1111 (1996)

8. Cheng, C, Xu, J: Boundedness of solutions for a second-order differential equations. Nonlinear Anal. 7, 1993-2004 (2008)

9. Liu, B: Quasiperiodic solutions of semilinear Lienard reversible oscillators. Discrete Contin. Dyn. Syst. 12, 137-160 (2005)

10. Ortega, R: Boundedness in a piecewise linear oscillator and a variant of the small twist theorem. Proc. Lond. Math. Soc. 79, 381-413 (1999)

11. Moser, J: On invariant curves of area-preserving mapping of an annulus. Nachr. Akad. Wiss. Gött. Math.-Phys. Kl. 1962, 1-20 (1962)

12. Moser, J: Convergent series expansions for quasi-periodic motions. Math. Ann. 169, 136-176 (1967)

13. Moser, J: Stable and Random Motions in Dynamical Systems. Princeton University Press, Princeton (1973)

10.1186/1687-1847-2014-55

Cite this article as: Wang and Jiang: Boundedness of solutions of forced isochronous oscillators with singularity at resonance. Advances in Difference Equations 2014, 2014:55

\section{Submit your manuscript to a SpringerOpen ${ }^{\circ}$ journal and benefit from:}

- Convenient online submission

Rigorous peer review

- Immediate publication on acceptance

- Open access: articles freely available online

- High visibility within the field

- Retaining the copyright to your article 